\title{
How to Prevent Recurrences in Gastric Cancer - Clinical Improvement by Multimodal Treatment?
}

\author{
H.-J. Meyer ${ }^{a} \quad$ H. Wilke ${ }^{b}$ \\ a Klinik für Allgemein- und Viszeralchirurgie, Städtisches Klinikum Solingen \\ b Klinik für Innere Medizin und Onkologie, Kliniken Essen-Mitte, Essen
}

\section{Introduction}

Gastric cancer is still a leading cause for mortality from malignant disease in the Western world, independent of changing epidemiological findings. Despite numerous perioperative procedures being available, surgery remains the cornerstone of potentially curative treatment performing $\mathrm{R} 0$ resection. Nevertheless, the majority of patients is diagnosed and treated with locally advanced or disseminated tumor stages; therefore, the risk for recurrence is even high after complete tumor resection [1-3].

\section{Patterns and Mechanisms of Recurrence and Metastases}

Although different ways of dissemination in gastric carcinoma are possible, i.e. direct invasion into other organs or via lymphatic and hematogenous routes, most of the cases show a combined pattern of recurrences. Based on clinical and autopsy studies, it could be demonstrated that local and/or intraperitoneal metastases are the only sites in early recurrence. Failures in the gastric bed, liver metastases or peritoneal carcinomatosis are found in about $50 \%$ of cases, whereas extraabdominal metastases without intraabdominal dissemination occur rarely. Even after potentially curative resection, more than $60 \%$ of failures are detected during the first two years after surgery. Intermediate recurrences within the 3rd and 5th postoperative year can be obtained in about one third of patients, and late recurrence after the 5 th postoperative year is an exception.

Following preoperative staging as well as pathohistological examinations, the incidence of lymph node metastases at the time of diagnosis can grow up to $60 \%$ and more, depending on the depth of tumor infiltration into the stomach well. In another $20-30 \%$ of patients, peritoneal metastases and liver metastases are detected by laparoscopic- or surgical exploration. Patterns of recurrence can be influenced by the histomorphological classification according to Lauren. For the intestinal type of cancer, liver metastases are more characteristic, whereas in diffuse-type peritoneal metastases can be found more often [3].
Despite new immunohistological or molecular techniques and increased knowledge about sophisticated prognostic factors, the mechanisms and reasons for locoregional recurrence are still under discussion. Besides limited surgical procedures, local relapses in the gastric bed and peritoneal cavity can arise from exfoliated tumor cells during surgical procedures, as shown in studies with examination of free cancer cells in the abdominal lavage before and after resection. The hypothesis of 'tumor cell entrapment' might be another important aspect in regard to the high incidence of early intraabdominal recurrences [3].

\section{Surgical Treatment}

Controversial discussion about the adequate extent of surgical resection is still ongoing, but the goal of the planned procedure must be complete tumor removal defined as R0-resection. $\mathrm{R} 0$-resection without any micro- and macroscopical residual tumor at all margins of the resected specimen is the most important prognostic factor after surgery, documented in numerous uni- and multivariate analyses.

Beside some cases of mucosa-type early gastric cancer which can be treated by different local methods endoscopically or with minimally invasive procedures, complete surgical resection of the tumor represents standard treatment. The resection lines at the stomach wall depend on the tumor site and the histomorphological classification described by Lauren. Taking into account the histological growth characteristics, the extent of resection at the stomach can be defined. The intestinal type requires a proximal margin of about $5 \mathrm{~cm}$, the diffuse type one of $8 \mathrm{~cm}$, measured in situ, to avoid intraluminal recurrence at the anastomotic site [1]. Taking into consideration these oral margins of clearance, subtotal distal and total gastrectomy are complementary procedures: in early gastric carcinoma of both types and in intestinal-type cancer localized in the distal half of the stomach subtotal distal gastrectomy is indicated. All other sites or types of gastric carcinoma require total or extended total gastrectomy including the resection of the distal esophagus.

The value of systematic lymphadenectomy (D2 dissection) in regard to the therapeutical benefit is once more under discussion after the performance of two randomized studies performed in

\begin{tabular}{ll}
\hline KARGER & (1) 1999 S. Karger GmbH, Freiburg \\
$\begin{array}{l}\text { Fax +49 761 4520714 } \\
\begin{array}{l}\text { E-mail kargergmbh@aol.com } \\
\text { www.karger.com }\end{array}\end{array}$ & Accessible online at: \\
http://BioMedNet.com/karger
\end{tabular}

Prof. Dr. med. H.-J. Meyer

Klinik für Allgemein- und Viszeralchirurgie

Städtisches Klinikum Solingen

Gotenstraße 1

D-42653 Solingen (Germany) 
the Netherlands and in the United Kingdom. The majority of retro- or prospective trials as carried out in Japan and the Western world could demonstrate improved survival rates, i.e. in stage II disease, compared to limited procedures. In contrast, both actual randomized studies pointed out significantly increased postoperative morbidity and mortality after extended lymph node dissection. But exact analysis of these trials showed that limited experiences with the performance of systematic lymphadenectomy - only one operation per center and year - exist; furthermore, in one of the studies obligatory splenectomy and resection of the tail of the pancreas was part of $\mathrm{D} 2$ resection.

Therefore, no survival prolongation could be obtained in these studies. In our opinion, systematic lymphadenectomy has to be integrated into the intended $\mathrm{R} 0$ resection. Following this procedure, an increased survival can be expected in case of limited numbers of metastatic lymph nodes, otherwise the incidence of locoregional recurrence might be decreased without influencing the occurrence of peritoneal carcinomatosis or distant metastases [1].

Nevertheless, unchanged poor long-term survival of advanced gastric cancer after surgical treatment alone and the high incidence of recurrent carcinomas has led to a more intense application of other modalities, as chemo- and/or radiotherapy carried out pre-, intra- or postoperatively.

\section{Preoperative Multimodality Treatment}

Complete tumor resection in locally advanced tumor stages (UICC stage III/IV) can be achieved in about $50 \%$ of cases. Therefore, several phase II/III studies with different preoperative chemotherapy regimens were performed in locally advanced as well as in potentially resectable gastric carcinomas. The aim of this modality was an intended reduction of locoregional tumor mass as well as destruction of occult systemic metastases. The majority of trials performed could show major response rates between 40 and $60 \%$ and more, resulting in a complete resection rate of the tumor of up to $80 \%$. Until now, a median survival rate of about $16-18$ months could be obtained; furthermore, about $10-20 \%$ of all patients are alive after the 5th postoperative year. Although the development of distant metastases could be delayed in some trials, the majority of patients died from problems caused by locoregional and intraperitoneal failures. Therefore, additional treatment options have to be investigated in the future $[1,2,4]$.

\section{Intraoperative Multimodality Treatment}

According to the high rate of locoregional failures even after preoperative treatment, different options of intraoperative modalities have been evaluated: intraoperative radiotherapy (IORT) as well as intraperitoneal chemotherapy alone or combined with hyperthermia or special carriers such as activated carbon particles.

The application of IORT proposes to increase the local dose to the tumor bed with the aim of improved local tumor control. Whereas one study could show survival benefit in stage II-IV disease [5], in contrast, other trials could obtain only a decrease of locoregional relapse but without any impact on distant tumor spreading or survival rates. Therefore, the value of IORT is discussed and the results obtained in different studies performed are limited and contradictory. This treatment is still an experimental modality and more trials are necessary to assess the potential value of such an approach [6].

To elevate administration of cytotoxic agents, intraperitoneal chemotherapy was carried out after complete tumor resection of locally advanced carcinomas. The aim of this procedure is the reduction of the incidence of peritoneal carcinomatosis, taking into account the hypothesis of tumor cell entrapment of exfoliated tumor cells during resective procedures. The data of some randomized studies could demonstrate a positive effect on the survival rates after intraperitoneal chemotherapy combined with hyperthermia. A significant decrease of peritoneal metastases could be found, but patterns of recurrence in regard to hepatic metastases or distant spreading could not be influenced. Furthermore, according to the results of these studies it was obvious that cytotoxic effects of different agents depend strongly on the time of administration. Positive results with decreased incidence of peritoneal metastases could only be obtained when intraperitoneal chemotherapy was performed intraoperatively or in the early postoperative phase. Otherwise, the positive impact of this procedure is limited or questionable $[7,8]$.

Taking into account the knowledge that free cancer cells are taken up only by lymphatic tissue in the peritoneum to develop metastases, chemotherapy was administrated by the application of mitomycin $\mathrm{C}$ bound to activated carbon particles which were taken up directly by the lymphatic tissue in the peritoneal cavity. Furthermore, the anticancer agent can be released for a longer period. Although some Asian trials showed improved survival rates after curative resection and adjuvant intraperitoneal treatment compared to surgery alone $[8,9]$, European trials failed to demonstrate any benefit following this procedure. In contrast, one phase III trial with significantly increased postoperative complications after this modality led to a breakdown of the study [10].

The combination of preoperative systematic chemotherapy followed by postoperative intraperitoneal application was also carried out to reduce the intraabdominal recurrent rate. Following two cycles of chemotherapy preoperatively, intraperitoneal chemotherapy was applied after curative resection in the $3 \mathrm{rd}$ for 4 th postoperative week. After a medium follow-up period of more than 3 years, the overall survival rates could be improved compared to historical controls, but other studies could obtain a positive impact only after intra- or early postoperative administration of intraperitoneal chemotherapy $[8,11]$.

\section{Adjuvant Postoperative Multimodality Treatment}

Adjuvant therapy after resection of gastric cancer was already carried out more than 20 years ago. Adjuvant chemotherapy applied early in the postoperative phase is a standard and basic treatment in Asia. Beside the systematic application of mitomycin $\mathrm{C}$ plus oral fluoropyriamidines, the combina- 
tion with immunotherapy using different agents could improve the disease-free - as well as the overall survival rates in different controlled clinical studies. Significant survival improvement could be obtained in some trials performing adjuvant intraperitoneal chemotherapy in the postoperative phase $[8,9,12]$.

In the Western world the adjuvant application of the chemotherapy combination FAM (fluorouracil, adriamycin, mitomycin) including several modifications was favored, administered 4 or 6 weeks postoperatively. But the vast majority of randomized trials and different metaanalyses of these studies could not show any positive impact on survival rates, and at present it must be stated that systemic adjuvant chemotherapy cannot be considered as a standard treatment in the Western hemisphere. The differences between Japanese and Western clinical trials of adjuvant chemotherapy can be explained by the administration of the drugs immediately after curative resection with a minimum of possible residual tumor cells - as performed in Japan. A better or more effective selection of the drugs used in Japanese trials seems to be questionable because more active and new regimens of adjuvant chemotherapy have been available worldwide in the last years. Therefore, taking into account the fact that gastric cancer is a chemosensitive tumor demonstrated by preoperative studies in locally advanced stages, well-designed protocols, i.e. patients suffering from high risk of early relapse, in the future are necessary and justified to investigate the possible positive impact of adjuvant chemotherapy even in Western countries $[13,14]$. After adjuvant radiotherapy, evaluated only in few studies, also no improvement of the survival rates could be obtained. One randomized study including more than 400 patients with surgical treatment alone or followed by adjuvant radio- or chemotherapy could not show any differences of the survival: the 5-year survival rate was 20,12 , or $19 \%$, respectively [15]. Therefore, the use of adjuvant modalities after surgical treatment especially in highrisk patients seems to be mandatory, but this procedure should be restricted only to controlled trials.

\section{Conclusion}

Complete surgical resection of gastric carcinoma is still the treatment of choice for gastric carcinoma. Despite improved and extended preoperative diagnostic procedures, the majority of patients suffer from locally advanced stages at the time of diagnosis. But after exact operative staging including endosonography and laparoscopy to exclude peritoneal dissemination and liver metastases, it is possible to find out patients in whom R0-resection is questionable. These patients should be treated with preoperative systemic chemotherapy to reduce the tumor mass and to enable complete tumor resection. But even after such a modality, the rate of locoregional relapses and peritoneal metastases is still high, although the development of distant metastases could be decreased. Because systematic adjuvant treatment could not show any therapeutical benefit until now, in the Western world additional intraperitoneal chemotherapy might be a promising approach. Nevertheless, some other aspects are of high interest, like selection of high-risk patients, prediction of possible response, choice of more effective cytotoxic agents, as well as timing and duration of the therapy. Furthermore, numerous biological as well as molecular prognostic parameters are still under investigation, but many problems concerning the clinical significance and prognostic value of those factors still remain. Future advances in molecular pathology of gastric cancer developing new tumor marker antibody systems shall lead to translation into clinical procedure, i.e. selection of patients for preoperative as well as for adjuvant treatment. Although surgical procedures could be standardized resulting in decreased postoperative mortality and increased resection rates, other treatment modalities are necessary to improve the overall survival rates. Different treatment options available can be carried out, but taking into account the individual situation of the patient and the tumor stage, those procedures must be tolerable and acceptable for the patient, and the management must be safe and uncomplicated.

\section{References}

1 Meyer H-J, Jähne J, Wilke H: Perspectives of surgery and multimodality treatment in gastric carcinoma. J Cancer Res Clin Oncol 1983;119:384-394.

2 Wilke H, Meyer H-J, Stahl M, Fink U: Aktueller Stand der neoadjuvanten Chemotherapie beim Magenkarzinom. Onkologe 1998;4:310-316.

3 Averbach AM, Jacquet P: Strategies to decrease the incidence of intraabdominal recurrence in resectable gastric cancer. Br J Surg 1996;83:726-733.

4 Meyer HJ, Opitz GJ, Jähne J, Wilke H: Hypothetisches und Gesichertes zur prae-, intra- und postoperativen Zusatztherapie des Magencarcinoms. Langenbecks Arch Chir Suppl II 1998;312-317.

5 Abe M, Takahashi M, Ono K, Tobe T, Inamoto T: Japan gastric trials in intraoperative radiation therapy. Int J Radiat Oncol Biol Phys 1998;15:1431-1433.

6 Budach VGF: The role of radiation therapy in the management of gastric cancer. Ann Oncol 1994;5: $37-48$.
7 Yonemura Y, Ninomiya I, Kaji M, Sugiyama K, Fujimura K, Sawa T, Katayama K, Tanaka S, Hirono Y, Miwa K, Miyazaki I: Prophylaxis with intraoperative chemohyperthermia against peritoneal recurrence of serosal invasion-positive gastric cancer. World J Surg 1995;19:450-455.

8 Sugarbaker PH, Averbach AM, Chang D: Adjuvant perioperative intraperitoneal chemotherapy for resectable gastric cancer; in Siewert JR, Roder JD (eds.): Progress in Gastric Cancer Research. Bologna, Monduzzi, 1997, pp 1399-1405.

9 Nakajima T: Adjuvant chemotherapy in gastric cancer. Japanese Approach; in Siewert JR, Roder JD (eds.): Progress in Gastric Cancer Research. Bologna, Monduzzi, 1997, pp 1451-1456.

10 Rosen HR, Jatzko G, Repse S, Potra S, Neudorfer H, Sandbichler P, Zacherl J, Rabl H, Holzberger P, Lisborg P, Czejka M: Adjuvant intraperitoneal chemotherapy with carbon-adsorbed mitomycin in patients with gastric cancer: Results of a randomized multicenter trial of the Austrian working group for surgical oncology. J Clin Oncol 1998;16:2733-2738.
11 Crookes P, Leichman CG, Leichman L, Tan M, Laine L, Stain S, Baranda J, Casagrande Y, Groshen S, Silberman H: Systematic chemotherapy for gastric carcinoma followed by postoperativ intraperitoneal therapy. Cancer 1997;79:1767-1775.

12 Nakazato H, Koike A, Saji S, Ogawa N, Sakamoto J: Efficacy of immunochemotherapy as adjuvant treatment after curative resection of gastric cancer. Lancet 1994;343:1122-1126.

13 Hermans J, Bonenkamp JJ, Boon MC, Bunt AMG, Ohyama S, Sasako M, van de Velde CJH: Adjuvant therapy after curative resection for gastric cancer: Metaanalysis of randomized trials. J Clin Oncol 1993;11:1441-1447.

14 Wils J: Future directions in the treatment of localized gastric cancer. A Western point of view; in Siewert JR, Roder JD (eds.): Progress in Gastric Cancer Research. Bologna, Monduzzi, 1997, pp 1441-1449.

15 Hallissey MT, Dunn JA, Ward LC, Allum WH: The second British Stomach Cancer Group trial of adjuvant radiotherapy or chemotherapy in resectable gastric cancer: Five-year follow-up. Lancet 1994;343: 1309-1312. 